\title{
Influência da concentração de condicionamento do ácido hidrofluorídrico na adesão protética de cerâmicas vítreas: uma revisão sistemática
}

The influence of hydrofluoric acid etching concentration on the prosthetic adlhesion of glass ceramics: a systematic review

Influencia de la concentración de acondicionamiento del ácido fluorhídrico en la adhesión de prótesis de cerámicas vítreas: una revisión sistemática

Recebido: 18/01/2021 | Revisado: 21/01/2021 | Aceito: 28/01/2021 | Publicado: 04/02/2021

\author{
Millena Silva Cardoso \\ ORCID: https://orcid.org/0000-0002-5414-6270 \\ Universidade Federal de Juiz de Fora, Brasil \\ E-mail: mscardoso.98@gmail.com \\ Laís Ferrante de Faria \\ ORCID: https://orcid.org/0000-0002-1094-0464 \\ Universidade Federal de Juiz de Fora, Brasil \\ E-mail: laisferrantedefaria@gmail.com \\ Camila Moreira Lima \\ ORCID: https://orcid.org/0000-0003-3233-5684 \\ Universidade Federal de Juiz de Fora, Brasil \\ E-mail: camila_moreira21@hotmail.com \\ Fabíola Pessôa Pereira Leite \\ ORCID: https://orcid.org/0000-0001-6316-5679 \\ Universidade Federal de Juiz de Fora, Brasil \\ E-mail: fabiola-leite1@hotmail.com \\ Laércio Almeida de Melo \\ ORCID: https://orcid.org/0000-0002-9276-0116 \\ Universidade Federal do Rio Grande do Norte, Brasil \\ E-mail: laercio_melo91@hotmail.com
}

\begin{abstract}
Resumo
Objetivo: O objetivo dessa revisão sistemática foi avaliar a influência das diferentes concentrações do ácido hidrofluorídrico (HF) no condicionamento das cerâmicas vítreas. Materiais e Métodos: Esta revisão reuniu estudos in vitro a partir de buscas nas bases Cochrane Library, Lilacs, PubMed, SciElo, Scopus e Web of Science, através dos descritores "Veneers", "Crowns", "Ceramics", "Ceramic", "Surface Treatments", "Surface", "Hydrofluoric Acid Concentration", "Acid Concentration", "Adhesion", "Hydrofluoric Acid". Foram incluídos estudos que investigaram os efeitos da concentração do ácido hidrofluorídrico através de diferentes concentrações entre grupos. O nível de evidência dos artigos selecionados seguiu os princípios do Oxford Center for Evidence-Based Medicine. Esta revisão seguiu as recomendações do PRISMA (Preferred Reporting Items for Systematic Reviews and Meta-analyzes). Resultados: As estratégias de busca encontraram 151 títulos, dos quais 8 foram eleitos e incluídos nessa revisão. Os parâmetros de avaliação incluíram a influência da concentração do ácido quanto a resistência a flexão, rugosidade superficial, ângulo de molhamento e resistência ao microcisalhamento. Nos estudos, as cerâmicas foram condicionadas com diferentes tipos de concentração e, no geral, o HF a 5\% teve o melhor desempenho. Conclusão: Os estudos encontraram mais resultados negativos para as concentrações mais baixas (1\% e 3\%). Apesar de algumas vantagens, o HF a $10 \%$ também não teve os melhores resultados, os quais foram de autoria do HF a 5\%. Além disso, a concentração não pode ser vista como o único e principal fator importante na qualidade de adesão das cerâmicas.

Palavras-chave: Condicionamento ácido do dente; Concentração de íons de hidrogênio; Ácido fluorídrico; Cerâmica; Coroas; Facetas dentárias; Adesividade; Propriedades de superfície.
\end{abstract}

\footnotetext{
Abstract

Aim: The aim of this systematic review is to evaluate the influence of the different concentrations of hydrofluoric acid (HF) on the etching of glass ceramics. Materials and Methods: This review searched in vitro studies on the Cochrane Library, Lilacs, PubMed, SciElo, Scopus and Web of Science, using the keywords "Veneers", "Crowns", "Ceramics", "Ceramic", "Surface Treatments", "Surface", "Acid Concentration", "Hydrofluoric Acid Concentration", "Adhesion", "Hydrofluoric Acid". Studies that investigated the effects of the HF concentration in groups with different concentrations were included. The level of evidence of the selected articles followed the principles of the Oxford Center for Evidence-Based Medicine. This review followed the recommendation of PRISMA (Preferred Reporting
} 
Items for Systematic Reviews and Meta-analyzes). In the studies, different types of concentration were used on the ceramics, and, in general, the 5\% HF concentration had the best performance. Results: The searching strategy found 151 titles, from which 8 were selected and included in the review. The standards of evaluation included the influence of acid concentration in flexural resistance, surface roughness, contact angle and micro shear resistance. Conclusion: The studies found more negative results in lower concentrations (1\% and 3\%). In spite of some advantages, the $10 \%$ $\mathrm{HF}$ also did not present the best results, when compared to 5\% HF. Moreover, the concentration should not be seen as the only and most important factor of the quality of ceramics adhesion.

Keywords: Acid etching dental; Hydrogen-Ion concentration; Hydrofluoric acid; Ceramic; Crowns; Dental Veneers; Adhesiveness; Surface properties.

\section{Resumen}

Objetivo: El objetivo de esta revisión sistemática fue evaluar la influencia de las diferentes concentraciones de ácido fluorhídrico (HF) en el acondicionamiento de las cerámicas vítreas. Materiales y Métodos: Por medio de esta revisión, se reunieron estudios in vitro a partir de búsquedas en las bases Cochrane Library, Lilacs, PubMed, SciElo, Scopus y Web of Science, a través de los filtros "Adhesion", "Ceramic", "Ceramics", "Crowns", "Hydrofluoric Acid", "Hydrofluoric Acid Concentration"”, "Acid Concentration" "Surface", "Surface Treatments", "Veneers". Fueron considerados estudios que investigan los efectos de la concentración de ácido fluorhídrico a través de diferentes concentraciones entre grupos. Para la búsqueda de evidencias en los artículos seleccionados se siguieron los principios del Oxford Center for Evidence-Based Medicine. Además, en esta revisión se adoptaron las recomendaciones de PRISMA (Preferred Reporting Items for Systematic Reviews and Meta-analyzes). Resultados: Por medio de las estrategias de búsqueda se encontraron 151 títulos, de los cuales fueron seleccionados 8 e incluidos en la revisión. Como parámetros de evaluación, se incluyeron la influencia de la concentración del ácido en relación a resistencia, flexión, rugosidad superficial, ángulo de contacto y resistencia al microcizallamiento. En los estudios, las cerámicas fueron acondicionadas con diferentes tipos de concentración y, por lo general, la de HF a 5\% tuvo mejor desempeño. Conclusión: En los estudios se encontraron más resultados negativos para las concentraciones más bajas (1\% y 3\%). A pesar de existir algunas ventajas, el HF a 10\% tampoco tuvo mejores resultados. Estos últimos se encontraron en HF a 5\%. Además, la concentración no puede ser vista como el único ni principal factor importante en la calidad de adhesión de las cerámicas.

Palabras clave: Grabado ácido dental; Concentration de iones de hidrógeno; Ácido fluorhídrico; Cerámica; Coronas; Coronas com frente estético; Adhesividad; Propriedades de superficie.

\section{Introdução}

O ácido hidrofluorídrico (HF) é usualmente utilizado como protocolo no tratamento das cerâmicas vítreas odontológicas devido a sua capacidade de garantir um preparo eficiente da superfície da peça que será cimentada (PuppinRontani et al., 2017). Esse tratamento inclui tanto uma interação química com a superfície da cerâmica vítrea, quanto mecânica, por meio do embricamento do agente de união (silano) usualmente utilizado e do cimento resinoso (Saracoglu et al., 2011; Veríssimo et al., 2019). Apesar do conhecimento de seu mecanismo de ação, não há atualmente um consenso quanto ao protocolo de condicionamento desse ácido (Mokhtarpour et al., 2017; Saracoglu et al., 2011). Essas variações de protocolo estão presentes na concentração e no tempo de aplicação do HF, o que pode influenciar nas propriedades mecânicas e adesivas da cerâmica (Vila-Nova et al., 2019).

Essa dependência quanto aos fatores tempo e concentração tem, inclusive, justificado a descoberta dos diversos estudos que apontam o HF, principalmente quando em altas concentrações e tempo de aplicação longo, como um potencial agente no enfraquecimento das cerâmicas (Miranda et al., 2019; Mokhtarpour, Alaghehmand \& Khafri, 2017; Moura et al., 2020; Venturini, Prochnow, May, et al. 2018). Concomitantemente com os outros procedimentos realizados durante a cimentação final, ele seria um fator comprometedor da resistência mecânica e do desempenho adesivo das peças, promovendo fraturas ou perdas de retenção (Souza \& Bottino, 2018). Também é de grande relevância citar o potencial danoso e agressivo do ácido hidrofluorídrico aos tecidos moles (Prochnow, Venturini, Scherer, et al. 2018; Venturini et al., 2018), desvantagem que tem ligação com a concentração do mesmo. A concentração é apontada, inclusive, como um dos parâmetros principais para determinação do padrão de penetração do ácido (Özean et al., 2012) sendo que, com o aumento da concentração, há um dano mais extenso e profundo dos tecidos, promovendo necrose tecidual e descalcificações ósseas (Dennerlein et al., 2016).

O ácido hidrofluorídrico a uma concentração de 10\%, apesar de ser um dos mais usuais no mercado (Souza \& Bottino, 
2018), pode apresentar um desempenho exacerbado quanto à dissolução da matriz de esmalte, sendo, portanto, causador do enfraquecimento da cerâmica (Gómes et al., 2018; Prochnow, Venturini, Scherer, et al. 2018). O ácido a uma concentração de $5 \%$, por sua vez, também um dos mais usados no mercado (Souza \& Bottino, 2018), pode não apresentar uma micrografia superficial que permite a penetração homogênea do cimento nas irregularidades criadas (Venturini, Prochnow, May, et al. 2018). Em contrapartida, há pesquisas que também não encontram relevâncias para o desempenho mecânico e adesivo das cerâmicas mediante a alteração da concentração do ácido hidrofluorídrico (Colombo et al., 2019; Missau et al., 2018; Prochnow, Venturini, Guilardi, et al. 2018).

Diante as incertezas quanto a influência da concentração ácida na qualidade de adesão dos materiais protéticos, esta revisão sistemática de literatura se propôs a verificar as influências que podem ser promovidas por meio das alterações na concentração do HF na adesão de cerâmicas vítreas.

\section{Metodologia}

Essa revisão seguiu os critérios do Preferred Reporting Items for Systematic Reviews and Meta-analyses (PRISMA, 2009). O questionamento a ser estudado foi: "Qual a influência da concentração do ácido hidrofluorídrico na adesão de peças cerâmicas vítreas?”. A metodologia realizada é embasada por trabalhos prévios publicados na literatura (Melo et al., 2020; Medeiros et al. 2019). Diante a amostra limitada de estudos que realizaram uma pesquisa exclusiva do fator "concentração" em relação à qualidade da adesão protética, não foi viável uma análise estatística e, portanto, um estudo qualitativo foi realizado (Pereira, et al. 2018).

\subsection{Critérios de Inclusão e Exclusão}

Foram incluídos nessa revisão: apenas estudos in vitro que apresentassem grupos de estudo determinados, de modo a variar e comparar as concentrações do ácido hidrofluorídrico, analisando sua influência na qualidade de adesão das cerâmicas. Incluiu-se apenas estudos realizados em cerâmicas vítreas. Foram excluídos estudos que comparavam outros fatores fora a concentração do ácido hidrofluorídrico, além daqueles que não realizavam estudos comparativos ou que não possuíam grupos de intervenção.

\subsection{Estratégia de Busca}

Três revisores (M.S.C., L.F.F., C.M.L.) conduziram as pesquisas nas seguintes bases de dados eletrônicas: MedlinePubMed, Scopus, Lilacs, Web of Science e Cochrane Library. A partir dos descritores: "Hydrofluoric acid", "Adhesion", "Hydrofluoric Acid Concentration or Acid Concentration", "Surface Treatments or Surface" e "Veneers or Crowns or Ceramics or Ceramic", como demonstrado no Quadro 1. 
Quadro 1 - Estratégias de Busca nas Bases de Dados

\begin{tabular}{|c|c|}
\hline Database & Estratégia de Busca \\
\hline PUBMED & $\begin{array}{l}\text { ((((veneers OR crowns OR ceramics OR } \\
\text { ceramic) AND (surface treatments OR } \\
\text { surface)) AND (hydrofluoridric acid } \\
\text { concentration OR acid concentration)) AND } \\
\text { (adhesion)) AND (hydrofluoric acid) }\end{array}$ \\
\hline SCIELO & $\begin{array}{l}\text { "veneers" OR "crowns" OR "ceramics" OR } \\
\text { "ceramic" AND " surface treatments" OR } \\
\text { "surface" AND "hydrofluoridric acid } \\
\text { concentration" OR "acid concentration" } \\
\text { AND "adhesion" AND "hydrofluoric acid" }\end{array}$ \\
\hline SCOPUS & $\begin{array}{c}\text { ALL("Veneers" OR "Crowns" OR } \\
\text { "Ceramics" OR " Ceramic" ) AND } \\
\text { ALL(" Surface Treatments" OR "Surface") } \\
\text { AND ALL("Hydrofluoridric Acid } \\
\text { Concentration" OR "A cid Concentration" ) } \\
\text { AND ALL("Adhesion") AND } \\
\text { ALL("Hydrofluoric A cid" ) }\end{array}$ \\
\hline WEB OF SCIENCE & $\begin{array}{c}\text { TS }=(\text { veneers OR crowns OR ceramics OR } \\
\text { ceramic) AND TS }=\text { (surface treatments OR } \\
\text { surface) AND TS=(hydrofluoridric acid } \\
\text { concentration OR acid concentration) AND } \\
\text { TS=(adhesion) AND TS=(hydrofluoric acid) }\end{array}$ \\
\hline LILACS & $\begin{array}{l}\text { "veneers" OR "crowns" OR "ceramics" OR } \\
\text { "ceramic" AND " surface treatments" OR } \\
\text { "surface" AND "hydrofluoridric acid } \\
\text { concentration" OR "acid concentration" } \\
\text { AND "adhesion" AND "hydrofluoric acid" }\end{array}$ \\
\hline COCHRANE & $\begin{array}{l}\text { (veneers OR crowns OR ceramics OR } \\
\text { ceramic) AND (surface treatments OR } \\
\text { surface) AND (hydrofluoridric acid } \\
\text { concentration OR acid concentration) AND } \\
\text { (adhesion) AND (hydrofluoric acid) }\end{array}$ \\
\hline
\end{tabular}

Fonte: Autores (2020)

A partir do Quadro 1, pode-se perceber, portanto, que a pesquisa foi realizada em cada uma das bases de dados, seguindo as estratégias apropriadas para cada uma.

\subsection{Seleção dos Estudos e Coletas de Dados}

Após as buscas nas bases de dados, os títulos foram selecionados e organizados, como demonstrado no Fluxograma 1. 


\section{Fluxograma 1:}

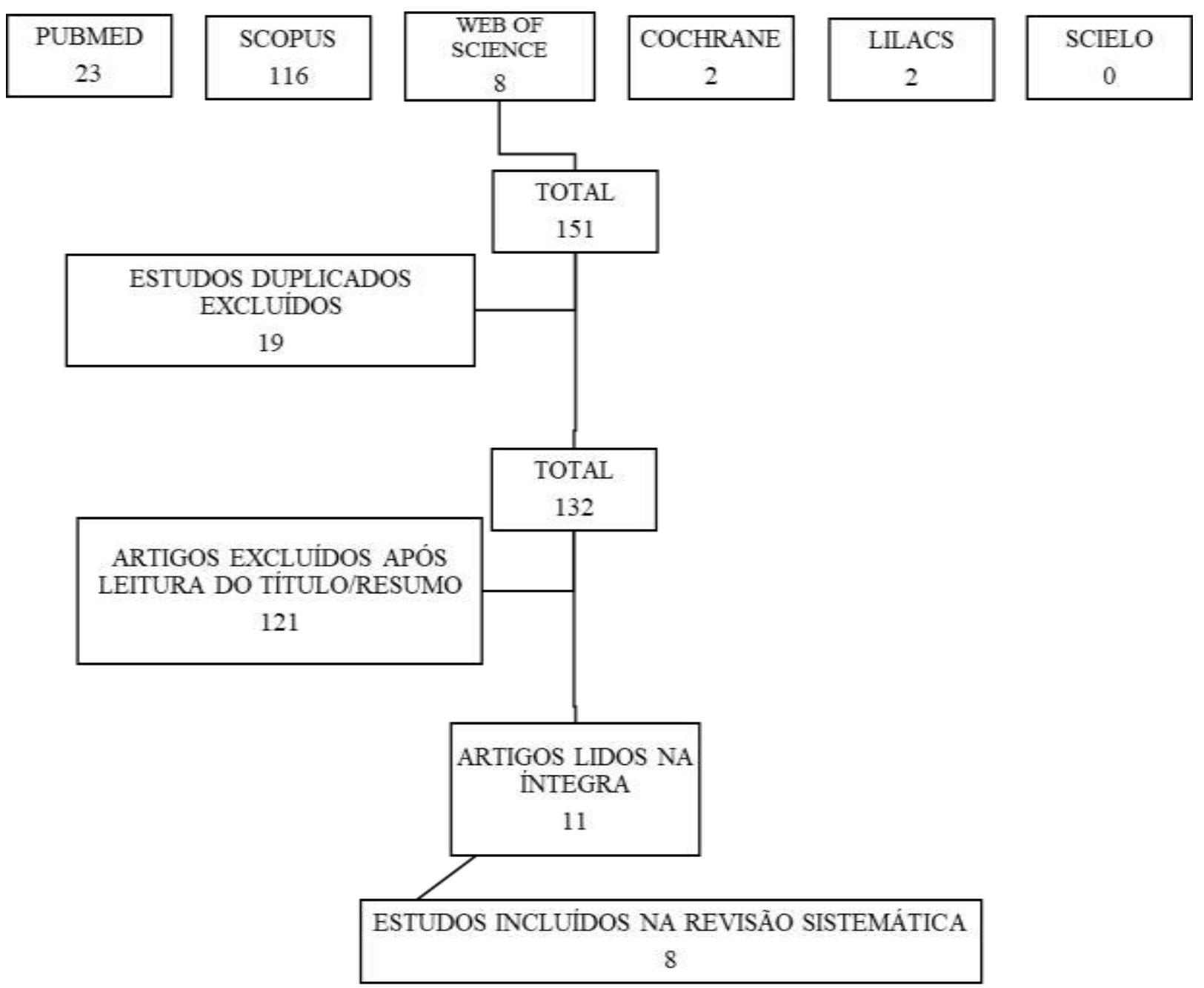

Fonte: Autores (2020)

Como demonstrado no diagrama, inicialmente excluiu-se os estudos duplicados. Os resumos dos artigos restantes foram lidos e, tomando como base os critérios de inclusão e exclusão, estipulou-se quais deles seriam lidos na íntegra e possivelmente incluídos na revisão. A partir de uma análise detalhada dos artigos lidos, junto com os critérios de seleção, determinou-se os estudos que seriam definitivamente incluídos nessa revisão.

Os artigos lidos em sua integralidade foram analizados conforme demonstra o Quadro 2. 
Quadro 2 - Análise dos Estudos Incluídos na Revisão

\begin{tabular}{|c|c|c|c|c|c|c|c|c|}
\hline Estudo & Referência & Local & $\begin{array}{c}\text { Tipo } \\
\text { de } \\
\text { Estudo }\end{array}$ & $\begin{array}{l}\text { Grupo de } \\
\text { Estudo }\end{array}$ & Amostras & $\begin{array}{l}\text { Parâmetros } \\
\text { Avaliados }\end{array}$ & $\begin{array}{l}\text { Tipo de } \\
\text { cerâmica }\end{array}$ & $\begin{array}{l}\text { Principais } \\
\text { Resultados }\end{array}$ \\
\hline $\begin{array}{l}\text { Prochnow } \\
\text { et al, } \\
2018\end{array}$ & 14 & $\begin{array}{c}\text { Rio } \\
\text { Grande } \\
\text { do Sul } \\
\text {-Brasil }\end{array}$ & $\begin{array}{l}\text { In- } \\
\text { vitro }\end{array}$ & $\begin{array}{c}\text { Grupos foram } \\
\text { separados de } \\
\text { acordo com as } \\
\text { diferentes } \\
\text { concentrações do } \\
\text { HF de } 1 \%, 3 \% \text {, } \\
5 \% \text { e } 10 \%\end{array}$ & $\begin{array}{l}\text { Para estudo do } \\
\text { ângulo de contato: } \\
\mathrm{n}=25 \text { peças de } \\
\text { cerâmica (5 grupos } \\
\text { com } \mathrm{n}=5 \text {. Cada } \\
\text { grupo uma } \\
\text { concentração }+ \\
\text { controle sem } \\
\text { condicionamento). } \\
\text { Para estudo do } \\
\text { microcisal hamento } \\
\mathrm{n}=80 \text { blocos de } \\
\text { cerâmica( } 8 \text { grupos } \\
\text { com n=10. Cada } \\
\text { grupo com uma \% } \\
\text { em condições } \\
\text { básicas e de } \\
\text { envelhecimento). }\end{array}$ & $\begin{array}{l}\text { A adesão foi } \\
\text { avaliada por meio: } \\
\text { do ângulo de } \\
\text { contato (técrica da } \\
\text { gota séssil); teste de } \\
\text { microcisalham ento } \\
\text { (EMC DL-2000); } \\
\text { do padrão de fratura } \\
\text { por meio da } \\
\text { esteromicroscopia; } \\
\text { da microestrutura } \\
\text { da superficie por } \\
\text { meio de } \\
\text { microscopia } \\
\text { eletrônica e a } \\
\text { rugosidade da } \\
\text { superficie por meio } \\
\text { do dispositivo } \\
\text { AFM }\end{array}$ & $\begin{array}{l}\text { Dissilicato de } \\
\text { Litio - IPS } \\
\text { e.Max CAD } \\
\text { (Ivoclar } \\
\text { Vivadent). }\end{array}$ & $\begin{array}{l}\text { O grupo controle e o } \\
\text { condicionado com } \\
\text { HF } 1 \% \text { apresentaram } \\
\text { os mai ores ângulos } \\
\text { de contato; no } \\
\text { microccisalham ento, } \\
\text { o envel hecimento } \\
\text { dem onstrou } \\
\text { prejudicar os val ores } \\
\text { de adesão. Os testes } \\
\text { de fratura não } \\
\text { apresentaram } \\
\text { alterações entre os } \\
\text { grupos; na análise de } \\
\text { superficie mai ores } \\
\text { irregularidades foram } \\
\text { encontradas nos } \\
\text { grupos de HF } 5 \% \mathrm{e} \\
10 \%, \text { que também } \\
\text { mostraram mai ores } \\
\text { rugosidades. }\end{array}$ \\
\hline $\begin{array}{l}\text { Venturini } \\
\text { et al, } \\
\mathbf{2 0 1 5}\end{array}$ & 25 & $\begin{array}{l}\text { Rio } \\
\text { Grande } \\
\text { do Sul- } \\
\text { Brasil }\end{array}$ & $\begin{array}{l}\text { In- } \\
\text { vitro }\end{array}$ & $\begin{array}{l}\text { Os grupos foram } \\
\text { separados de } \\
\text { acordo com as } \\
\text { variações na } \\
\text { concentração do } \\
\text { ácido fluoridrico } \\
(1 \%, 3 \%, 5 \%, \\
10 \%) \text { mais um } \\
\text { grupo controle } \\
\text { sem receber } \\
\text { tratamento }\end{array}$ & $\begin{array}{c}\text { Para análise do } \\
\text { ângulo de contato: } \\
n=25 \text { discos de } \\
\text { cerâmica (5 grupos } \\
\text { com } n=5 \text {. } \\
\text { Força de união a } \\
\text { microtração: } n=40 \\
\text { blocos de cerâmica } \\
(4 \text { grupos com } \\
n=10)\end{array}$ & $\begin{array}{l}\text { A adesão foi } \\
\text { medida a partir dos } \\
\text { fatores: ângul o de } \\
\text { contato (técrnica da } \\
\text { gota séssil); análise } \\
\text { da microtopografia } \\
\text { (SEM/AFM); } \\
\text { microtr ação com e } \\
\text { sem condições de } \\
\text { envelhecimento }\end{array}$ & $\begin{array}{l}\text { Fel dspática- } \\
\text { VITA Mark II } \\
\text { Cerec/inL ab, } \\
\text { 2MDC/ } \\
\text { 112, Vita } \\
\text { Zahnfabrik; } \\
\text { Bad } \\
\text { Säckingen, } \\
\text { Germany. }\end{array}$ & $\begin{array}{l}\text { A diminui ção do } \\
\text { ângulo de contato } \\
\text { correspondeu ao } \\
\text { aumento da } \\
\text { concentração do } \\
\text { áci do fluoridrico; } \\
\text { maiores } \\
\text { concentrações } \\
\text { geraram fossas e } \\
\text { crateras mais fundas; } \\
\text { o condicionamento e } \\
\text { suas diferentes } \\
\text { concentrações foram } \\
\text { estatisticam ente } \\
\text { significativos na } \\
\text { força de adesão } \\
\text { apenas após } \\
\text { envelhecimento. As } \\
\text { concentrações de } 3 \% \text {, } \\
5 \% \text { e } 10 \% \text { foram } \\
\text { igualm ente indicadas. }\end{array}$ \\
\hline $\begin{array}{l}\text { Venturini } \\
\text { et al, } \\
\mathbf{2 0 1 8}\end{array}$ & 23 & $\begin{array}{l}\text { Santa } \\
\text { Maria } \\
\text { (RS - } \\
\text { Brasil) }\end{array}$ & $\begin{array}{l}\text { In- } \\
\text { vitro }\end{array}$ & $\begin{array}{l}\text { Os grupos de } \\
\text { estudo foram } \\
\text { divididos } \\
\text { conforme as } \\
\text { diferentes } \\
\text { concentrações do } \\
\text { ácido fluoridrico } \\
(1 \%, 5 \% \text { e } 10 \%) \text {, } \\
\text { além de um } \\
\text { grupo controle } \\
\text { não condicionado }\end{array}$ & $\begin{array}{c}\mathrm{N}=80(4 \text { grupos } \\
\text { com } \mathrm{n}=20) \text { peças } \\
\text { cerâmicas em } \\
\mathrm{CAD} / \mathrm{CAM}\end{array}$ & $\begin{array}{l}\text { A adesão fơ } \\
\text { avaliada por meio } \\
\text { de um teste de } \\
\text { carga até falha por } \\
\text { meio do método da } \\
\text { escada A sperficie } \\
\text { das cerâmicas foi } \\
\text { analisada por meio } \\
\text { do escaneamento } \\
\text { microscópico e as } \\
\text { fraturas por um } \\
\text { microscópio óptico. }\end{array}$ & $\begin{array}{c}\text { Fel dspática- } \\
\text { Vita Mark II } \\
\text { 4M2C/I12; } \\
\text { Vita } \\
\text { Zahnfabrik. }\end{array}$ & $\begin{array}{l}\text { As coroas } \\
\text { condicionadas com } \\
\text { HF } 5 \% \text { tiveram } \\
\text { menores cargas } \\
\text { médias de falha por } \\
\text { fadiga quando } \\
\text { comparada aos outros } \\
\text { grupos, os quais não } \\
\text { obtiveram variações } \\
\text { entre si. Mais } \\
\text { crateras apareceram } \\
\text { com as maiores \%. }\end{array}$ \\
\hline $\begin{array}{l}\text { Missau et } \\
\text { al, } 2018\end{array}$ & 7 & $\begin{array}{l}\text { Santa } \\
\text { Maria } \\
\text { (RS- } \\
\text { Brasil) }\end{array}$ & $\begin{array}{l}\text { In- } \\
\text { vitro }\end{array}$ & $\begin{array}{l}\text { Os gnupos de } \\
\text { estudo foram } \\
\text { divididos } \\
\text { conforme as } \\
\text { diferentes } \\
\text { concentrações do } \\
\text { ácido fluoridrico } \\
1 \%, 5 \% \text { e } 10 \%\end{array}$ & $\begin{array}{c}\mathrm{N}=60 \text { pré molares } \\
\text { humanos }(3 \text { grupos } \\
\text { com } \mathrm{n}=20)\end{array}$ & $\begin{array}{l}\text { Preparos cavitários } \\
\text { do tipo inlay foram } \\
\text { padronizados e } \\
\text { realizadosnos } \\
\text { dentes. As peças em } \\
\text { cerâmicas foram } \\
\text { cimentadas sob } \\
\text { mesmo protocolo. } \\
\text { Realizou-se um } \\
\text { teste de carga de } \\
\text { falha por fadiga, } \\
\text { análise da } \\
\text { superficie e das } \\
\text { falhas. }\end{array}$ & $\begin{array}{c}\text { Fel dspática- } \\
\text { Vita Mark II } \\
\text { Cerec } \\
\text { inL ab, 2MDC / } \\
\text { I12, and } \\
2 \mathrm{MBC} / \mathrm{I1} 2 \\
\text { Vita } \\
\text { Zahnfabrik, } \\
\text { Bad } \\
\text { Säckingen, } \\
\text { Germanry. }\end{array}$ & $\begin{array}{l}\text { As concentrações não } \\
\text { influenciaram nas } \\
\text { cargas de falha sob } \\
\text { fadiga, no ponto de } \\
\text { vista estatistico. } \\
\text { Mai or parte das } \\
\text { falhas ocorreram na } \\
\text { interface dente- } \\
\text { resauração. } \\
\text { Diferentes } \\
\text { concentrações } \\
\text { alteraram a } \\
\text { micrografia da } \\
\text { superficie. }\end{array}$ \\
\hline
\end{tabular}




\begin{tabular}{|c|c|c|c|c|c|c|c|c|}
\hline $\begin{array}{l}\text { Prochnow } \\
\text { et al., } \\
2018\end{array}$ & 13 & $\begin{array}{l}\text { Santa } \\
\text { Maria } \\
\text { (RS- } \\
\text { Brasil) }\end{array}$ & $\underset{\text { vitro }}{\text { In- }}$ & $\begin{array}{c}\text { Formaram-se } 4 \\
\text { grupos } \\
\text { diferentes } \\
\text { ba seado nas } \\
\text { diferentes } \\
\text { concentra ções } \\
\text { do ácido } \\
\text { fluorídrico ( } 3,5 \\
\text { e } 10 \% \text { ) e um } \\
\text { grupo contro le } \\
\text { sem } \\
\text { condicionamento }\end{array}$ & $\begin{array}{c}\mathrm{N}=\mathbf{2 0} \text { discos de } \\
\text { cerâmica }(4 \\
\text { grupos com } \mathrm{n}=\mathbf{5})\end{array}$ & $\begin{array}{l}\text { Os grupos foram } \\
\text { submetidos a um } \\
\text { teste de cargas } \\
\text { cíclicas até } \\
\text { falla, por m eio do } \\
\text { método da escada. } \\
\text { A análise } \\
\text { topográfica foi por } \\
\text { meio de um } \\
\text { escaneamento com } \\
\text { microscopia } \\
\text { eletrônica e as } \\
\text { análises de fratura } \\
\text { por meio de } \\
\text { estereomicroscopia }\end{array}$ & $\begin{array}{c}\text { Dissilicato de } \\
\text { lítio- } \\
\text { IPS e.Max } \\
\text { CAD, Ivo clar } \\
\text { Viradent, } \\
\text { Schaan, } \\
\text { Liechtenstein. }\end{array}$ & $\begin{array}{c}\text { Os maiores valores } \\
\text { de carga foram } \\
\text { encontrados nos } \\
\text { condicionam entos } \\
\text { de } 3 \text { e } 5 \% \text { A análise } \\
\text { topográfica } \\
\text { demonstrou que } \\
\text { quando maior a } \\
\text { concentração do } \\
\text { HF, melhor é a } \\
\text { disso lução da matriz } \\
\text { vítria sendo as } \\
\text { maiores } \\
\text { encontradas no } \\
\text { grupo de HF5 e } \\
10 \% \text { As falhas } \\
\text { foram iniciadas } \\
\text { como fraturas } \\
\text { radiais partindo da } \\
\text { superficie de } \\
\text { cim entação. }\end{array}$ \\
\hline $\begin{array}{l}\text { Prochnow et } \\
\text { al, 2018 }\end{array}$ & 15 & $\begin{array}{l}\text { Santa } \\
\text { Maria } \\
\text { (RS- } \\
\text { Brasil) }\end{array}$ & $\begin{array}{l}\text { In- } \\
\text { vitro }\end{array}$ & $\begin{array}{l}\text { Os grupos foram } \\
\text { separados de } \\
\text { acordo com as } \\
\text { diferentes } \\
\text { concentraçóes do } \\
\text { ácido fluori drico } \\
\text { (HF): } 3 \%, 5 \% \text { e } \\
10 \% \text {, além de um } \\
\text { grupo controle } \\
\text { sem } \\
\text { condicionam ento }\end{array}$ & $\begin{array}{c}\mathrm{N}=75(4 \text { grupos } \\
\text { com } \mathrm{n}=18)\end{array}$ & $\begin{array}{l}\text { Após cimentados } \\
\text { em peças de } \\
\text { material análogo } \\
\text { dentina com } \\
\text { preparos protéticos } \\
\text { idênticos, as coroas } \\
\text { foram subm etidas a } \\
\text { teste de carga até } \\
\text { falha (técnica da } \\
\text { escada) e a análise } \\
\text { topográfica } \\
\text { (microscopia } \\
\text { eletrônica) e das } \\
\text { fraturas } \\
\text { (esteroscopia) }\end{array}$ & $\begin{array}{l}\text { Dissilicato de } \\
\text { litio- } \\
\text { IPS e.Max } \\
\text { CAD (Ivoclar } \\
\text { Vivadent). }\end{array}$ & $\begin{array}{l}\text { As concentrações } \\
\text { diferentes de HF não } \\
\text { afetam na fadiga das } \\
\text { coroas e as análises de } \\
\text { superficie mostraram } \\
\text { que a usinagem } \\
\text { predomina na influência } \\
\text { no relevo da superficie } \\
\text { formada, } \\
\text { independentem ente da } \\
\text { concentração ácida. }\end{array}$ \\
\hline $\begin{array}{l}\text { Venturini et } \\
\text { al, } 2015\end{array}$ & 22 & $\begin{array}{l}\text { Santa } \\
\text { Maria } \\
\text { (RS - } \\
\text { Brasil) }\end{array}$ & $\begin{array}{l}\text { In- } \\
\text { vitro }\end{array}$ & $\begin{array}{l}\text { Os grupos foram } \\
\text { divididos de } \\
\text { acordo com as } \\
\text { diferentes } \\
\text { concentrações dp } \\
\text { ácido fluoridrico } \\
\text { (HF): } 1 \%, 3 \%, \\
5 \% \text { e } 10 \% \text {, alem } \\
\text { de um grupo } \\
\text { controle sem } \\
\text { condicionamento. }\end{array}$ & $\begin{array}{c}\mathrm{N}=150 \text { barras de } \\
\text { cerâmica }(5 \text { grupos } \\
\text { cơm } \mathrm{n}=30)\end{array}$ & $\begin{array}{l}\text { A análise da } \\
\text { rugosidade da } \\
\text { superficie fơ feita } \\
\text { por meio de um } \\
\text { perfil ôm etro, a } \\
\text { força flexural foi } \\
\text { estudada a partir do } \\
\text { teste da curva de } \\
\text { três pontos, a } \\
\text { análise das fraturas } \\
\text { e topográfica por } \\
\text { meio de } \\
\text { microscopia } \\
\text { eletrônica }\end{array}$ & $\begin{array}{c}\text { Feldspática- } \\
\text { VitaBlocks } \\
\text { Mark II for } \\
\text { CEREC/inL ab, } \\
\text { Vita } \\
\text { Zahrfabrik, } \\
\text { Bad } \\
\text { Säckingen, } \\
\text { Germany. }\end{array}$ & $\begin{array}{c}\text { O aum ento da } \\
\text { concentração do HF } \\
\text { foi diretam ente } \\
\text { proporcional ao } \\
\text { aumento da } \\
\text { rugosidade da } \\
\text { cerâmica. Não houve } \\
\text { diferenças } \\
\text { significativas na força } \\
\text { flexural entre os } \\
\text { gupuos de diferentes } \\
\text { concentrações, apesar } \\
\text { do condicionamento } \\
\text { ácido ter reduzido a } \\
\text { força da cerâmica. }\end{array}$ \\
\hline $\begin{array}{l}\text { Venturini et } \\
\text { al, } 2018\end{array}$ & 24 & $\begin{array}{l}\text { Santa } \\
\text { Maria } \\
\text { (RS) - } \\
\text { Brasil }\end{array}$ & $\begin{array}{l}\text { In- } \\
\text { vitro }\end{array}$ & $\begin{array}{l}\text { Os grupos foram } \\
\text { separados de } \\
\text { acordo com os } \\
\text { diferentes tipos } \\
\text { de concentração } \\
\text { do ácido } \\
\text { fluoridrico: } 1 \% \text {, } \\
5 \% \text { e } 10 \% \text { além } \\
\text { de um grupo } \\
\text { controle sem } \\
\text { condicionam ento }\end{array}$ & $\begin{array}{c}\mathrm{N}=80 \text { discos de } \\
\text { cerâmica }(4 \text { grupos } \\
\text { cơm } \mathrm{n}=10)\end{array}$ & $\begin{array}{l}\text { Após cim entação } \\
\text { os grupos forma } \\
\text { submetidos a testes } \\
\text { de fadiga, por meio } \\
\text { do ex perimento da } \\
\text { escada Realiz ou-se } \\
\text { análise das fraturas } \\
\text { por meio do } \\
\text { microscópio optico } \\
\text { e análises } \\
\text { topográficas pela } \\
\text { microscopia } \\
\text { el etrônica. }\end{array}$ & $\begin{array}{c}\text { Feldspática- } \\
\text { VitaBlocks } \\
\text { Mark II for } \\
\text { CEREC finL ab, } \\
\text { Vita } \\
\text { Zahrfabrik, } \\
\text { Bad } \\
\text { Säckingen, } \\
\text { Germany. }\end{array}$ & $\begin{array}{c}\text { Os discos } \\
\text { condicionados com } \\
\text { ácido fluoridrico } 5 \% \\
\text { apresentados os } \\
\text { menores valores de } \\
\text { fadiga sob carga } \\
\text { quando comparado aos } \\
\text { outros grupos. As } \\
\text { fissuras promovidas } \\
\text { pelas concentrações de } \\
1 \text { e } 5 \% \text { não foram } \\
\text { totalmente preenchidas } \\
\text { com o cimento } \\
\text { resinoso. Asfraturas } \\
\text { se iniciaram do centro } \\
\text { da superficie } \\
\text { cimentada }\end{array}$ \\
\hline
\end{tabular}

Fonte: Elaborado pelo autor (2020)

Pode-se perceber, portanto, que os estudos incluídos nessa revisão foram registrados e analisados quanto aos autores, ao local onde foi realizada cada pesquisa, o tipo de estudo, o tamanho e divisão das amostras, a metodologia, o tipo de cerâmica vítrea utilizada e os principais resultados. 


\subsection{Avaliação do Risco de Viés}

A evidência dos artigos selecionados foi feita com base no Oxford Center for Evidence-Based Medicine (2011), como demonstra o Quadro 3.

Quadro 3. Classificação do Nível de Evidência.

\begin{tabular}{|c|c|c|c|}
\hline \multicolumn{4}{|c|}{ Nível de Evidência } \\
\hline Estudo & Referência & Grau de Recomendação & Nível de Evidência \\
\hline $\begin{array}{l}\text { Prochnow, Venturini, } \\
\text { Grasel, et al. } 2018\end{array}$ & 14 & B & $2 \mathrm{~B}$ \\
\hline $\begin{array}{l}\text { Venturini, Prochnow, } \\
\text { Rambo, et al. } 2015\end{array}$ & 25 & B & $2 \mathrm{~B}$ \\
\hline $\begin{array}{l}\text { Venturini, Prochnow, } \\
\text { May, et al. } 2018\end{array}$ & 23 & $\mathrm{~B}$ & $2 \mathrm{~B}$ \\
\hline Missau et al., 2018 & 7 & $\mathrm{D}$ & $\mathrm{D}$ \\
\hline $\begin{array}{l}\text { Prochnow, Venturini, } \\
\text { Scherer, et al. } 2018\end{array}$ & 13 & $\mathrm{~B}$ & $2 \mathrm{~B}$ \\
\hline $\begin{array}{l}\text { Prochnow, Venturini, } \\
\text { Guilardi, et al. } 2018\end{array}$ & 15 & B & $2 \mathrm{~B}$ \\
\hline $\begin{array}{l}\text { Venturini, Prochnow, } \\
\text { May, et al. } 2015\end{array}$ & 22 & B & $2 \mathrm{~B}$ \\
\hline $\begin{array}{l}\text { Venturini, Prochnow, } \\
\text { Pereira, et al. } 2018\end{array}$ & 24 & B & $2 \mathrm{~B}$ \\
\hline
\end{tabular}

Fonte: Autores (2020)

Os estudos, como demonstrado no quadro, foram categorizados em 1a, 1b, 1c, 2a, 2b, 2c, 3a, 3b, 4 e 5 nív eis. Os Níveis de Evidência 1 (CEBM) estabelecem uma abordagem para retratar este processo diante diferentes tipos de perguntas: terapia ou prevenção, etiologia ou dano, prognóstico, diagnóstico, diagnóstico diferencial ou prevalência de sintomas estudo e análises econômicas e de decisão. Grande parte dos artigos incluídos foram avaliados e classificados como 2B, sendo que apenas um teve o grau de recomendação inicial mantido, tomando como base o desenho da metodologia e risco de viés do sistema Grading of Recommendations Assessment, Development and Evaluation (Grade, 2008).

\section{Resultados}

A busca eletrônica nas bases de dados resultou em um total de 151 artigos. Desse total, ao eliminar os estudos duplicados e aqueles que se encaixavam nos critérios de exclusão, obteve-se um total de 11 artigos para serem integralmente lidos. Diante a última análise, 8 artigos foram terminantemente incluídos nesse estudo. Foram analisados um total de 635 amostras de cerâmicas vítreas, incluindo cerâmicas de dissilicato de lítio e feldpspáticas. As peças foram condicionadas com diferentes tipos de concentração do ácido hidrofluorídrico. Dessa forma, dentre as pesquisas realizadas com a cerâmica feldspática, $40 \%$ delas preconizou o uso do $\mathrm{HF}$ a $10 \%, 40 \%$ indicou eficácia similar entre as concentrações de HF a 5\% e $10 \%$ e, em $20 \%$ dos estudos, o uso exclusivo do HF a $5 \%$ foi indicado. Por outro lado, dentre os estudos realizados nas cerâmicas de dissilicato de lítio, $90 \%$ indicou o uso do HF a 5\%. 10\% desses estudos não diferenciou os resultados quanto ao uso do HF a 
$10 \%$.

Foram realizados diferentes testes de resistência mecânica entre os artigos, incluindo testes de carga de falha por fadiga, resistência à força flexural e de microcisalhamento. Em alguns casos as peças foram submetidas a condições de armazenamento com o objetivo de observar o comportamento desses fatores diante o envelhecimento, como ocorre na prática clínica. A qualidade da adesão também foi discutida a partir de análises microscópicas da micrografia superfícial após o ataque ácido, além de testes de molhamento, ângulo de contato superfícial e padrão de fratura das cerâmicas.

Tomando como base o objetivo do estudo, que busca observar a influência das diferentes concentrações do ácido fluorídrico na adesão das cerâmicas, percebeu-se que o uso do ácido a uma concentração de 5\% ou $10 \%$ foi a principal indicação nos estudos selecionados, sendo, pelo menos um dos dois, citados em todos os artigos. Dos artigos analisados, $90 \%$ coincidiram em relação as desvantagens no uso do ácido hidrofluorídrico a $1 \%$, sendo essa concentração considerada ineficiente devido ao fato de proporcionar uniões adesivas fracas.

De acordo com Prochnow, Venturini, Grasel, et al. (2018), durante a análise da adesão, percebeu-se que essa propriedade foi prejudicada quando houve a influência do envelhecimento nas peças, apresentando resultados diferentes de acordo com o condicionamento ácido. O uso do HF a 5\% e a 10\% foi o mais indicado, levando em consideração os resultados que os mesmos promoveram na topografia da superfície cerâmica de dissilicato de lítio, apresentando grande importância na efetividade da adesão. Do mesmo modo, Venturini, Prochnow, Rambo, et al. (2015) encontraram mudanças significativas na adesão devido às diferentes concentrações apenas após o envelhecimento das amostras de cerâmica feldspática (p=0,0033). De acordo com os resultados, apesar do uso do ácido hidrofluorídrico a 10\% ter promovido o menor ângulo de contato entre os grupos, esse não foi um fator relevante, já que as concentrações apresentaram estabilidades similares quanto a força de adesão. Dessa forma, todas as concentrações foram indicadas equivalentemente.

Já em outro estudo, Venturini, Prochnow, May, et al. (2018) ao analisarem as cargas médias de falha por fadiga encontraram os menores valores de carga naquelas cerâmicas felspáticas condicionadas com o ácido hidrofluorídrico a $5 \%$. Devido a criação de topografias superficiais mais favoráveis e terem apresentado valores de carga similares, o uso tanto do ácido hidrofluoródrico a $1 \%$ quanto a $10 \%$ foi indicado. Menores valores em relação à resistência de fadiga, também foram encontrados nos grupos condicionados por 5\% em outro estudo de Venturini, Prochnow, Pereira, et al. (2018), no qual, ao analisarem o tratamento de superfície das cerâmicas feldspáticas, foi proposto o uso do ácido hidrofluorídrico a $10 \%$ devido ao melhor embricamento do cimento resinoso na superfície condicionada sob essa concentração ácida.

Prochnow, Venturini, Scherer, et al. (2018), por outro lado, encontraram altos valores de carga de falha por fadiga para o condicionamento com o ácido hidrofluorídrico a 3\% e 5\%, sendo portanto, os mais recomendados para o uso, levando em consideração a redução das qualidades mecânicas e adesivas encontradas nos testes após o condicionamento com o ácido a uma concentração de $10 \%$ em cerâmicas de dissilicato de lítio.

Já em estudos realizados com peças cerâmicas feldspáticas fresadas em CAD/CAM, como na pesquisa de Missau et al. (2018), não foram encontradas influências estatísticamente significativas entre os diferentes tipos de concentração e sua possível influência nas cargas de falha sob fadiga $(\mathrm{p}=0,14)$, apesar de alterarem a micrografia das peças. Em outro estudo com peças de dissilicato de lítio fresadas em CAD/CAM, Prochnow, Venturini, Guilardi, et al. (2018), além de não encontrarem diferenças nos desempenhos mecânico e adesivo das peças tratadas com diferentes tipos de concentração do ácido hidrofluorídrico, demonstraram que a própria usinagem tem grande relevância e influência no relevo da superfície formada e, consequentemente, no desempenho da adesividade cerâmica.

A similaridade de desempenho à força flexural de cerâmicas feldspáticas condicionadas com os diferentes tipos de ácido hidrofluorídrico também foi encontrado no estudo de Venturini, Prochnow, May, et al. (2015). Apesar dos resultados semelhantes, a pesquisa indica o uso das concentrações de 3\% e 5\% no tratamento das cerâmicas feldspáticas, levando em 
consideração os possíveis riscos aos tecidos moles e à cerâmica que ocorrem a partir do uso da concentração do ácido a $10 \%$. Atentando-se ao fato de que o condicionamento, apesar da sua importância na cimentação, prejudica, sim, a força da cerâmica.

\section{Discussão}

Essa revisão teve como objetivo avaliar as evidências científicas presentes na literatura a respeito das influências que as diferentes concentrações do HF podem promover na adesão de peças cerâmicas vítreas com finalidades protéticas. Todos os estudos selecionados consistiram em pesquisas laboratoriais in vitro, nas quais haviam grupos de estudo distintos que permitissem uma análise comparativa entre o uso dos diferentes tipos de concentração ácida. A garantia quanto a validade desses estudos, assim como sua reprodutibilidade, foi avaliada por meio dos critérios de avaliação do nível de evidência do Oxford Center for Evidence-Based Medicine (2011).

As concentrações muito baixas não promoveram mudanças suficientes para favorecer uma boa adesão e, do mesmo modo, as concentrações muito altas, como a de $10 \%$, indicaram resultados negativos em algumas pesquisas, uma vez que apresentaram indícios de enfraquecimento em alguns tipos de cerâmica, principalmente nos estudos com dissilicato de lítio, e de alterações superficiais desvantajosas. Apesar dos testes para análise da adesão não terem sido os mesmos em todos os trabalhos, assim como as cerâmicas utilizadas em cada um, as buscas realizadas permitiram a seleção de oito estudos que apresentaram metodologias similares, assim como objetivos e parâmetros de avaliação coniventes com o objetivo dessa revisão.

O condicionamento com HF é o usual protocolo clínico para o tratamento de superfícies das peças cerâmicas vítreas, seguido pela aplicação de um agente de união (Moura et al., 2019). O HF é um importante passo para garantir tanto a adesão do cimento resinoso, quanto para assegurar a resistência mecânica do conjunto protético (Vila-Nova et al., 2019), uma vez que promoverá a dissolução da matriz vítrea, expondo sua cadeia polimérica que irá interagir com o agente de união, além de gerar porosidades, sulcos e irregularidades que auxiliam na força de ligação do silano e do cimento resinoso à cerâmica (Miranda et al., 2019; Sudré et al., 2020). Apesar da sua eficiência, atualmente não há um consenso quanto ao protocolo de condicionamento, levando em consideração as diferentes concentrações presentes no mercado e os mais variados tipos de materiais disponíveis (Veríssimo et al., 2019). Além dos diversos resultados clínicos que, dentre fraturas e perdas de adesão, ainda não se tem uma explicação definitiva quanto a influência que a concentração pode ter nessas falhas (Souza \& Bottino, 2018). Essa revisão sistemática, portanto, procurou avaliar as possíveis influências deste fator na adesão das cerâmicas.

Por meio da microscopia eletrônica, os estudos consentiram o fato do aumento da rugosidade superficial ser proporcional ao aumento da concentração, já que crateras mais largas e profundas aparecem sempre associadas a uma menor quantidade de matriz vítrea (Venturini, Prochnow, Pereira, et al. 2018). Essa rugosidade superficial é de grande relevância no embricamento do cimento resinoso já que, além da infiltração do mesmo nas porosidades e a criação de uma retenção mecânica, ela está associada ao aumento na energia livre de superfície e a redução do ângulo de contato entre esse produto e a região condicionada (Miranda et al., 2019; Sudré et al., 2020), fatores que auxiliam na infiltração e homogeneização do agente cimentante na cerâmica. O ângulo de contato é outro fator que, nas pesquisas em questão, apresentou um aumento proporcional à elevação da concentração do ácido hidrofluorídrico.

As pesquisas realizadas com o ácido a uma concentração de $1 \%$ demonstraram que as deformidades superficiais geradas por essa concentração foram pouco significativas ao compará-las ao grupo controle, que não recebeu condicionamento ácido (Venturini, Prochnow, May, et al. 2015; Venturini, Prochnow, Rambo, et al. 2015, Venturini, Prochnow, May, et al. 2018; Venturini, Prochnow, Pereira, et al. 2018), mesmo após um preparo bem realizado e o uso do silano (Prochnow, Venturini, Grasel, et al. 2018). Nesses estudos, a baixa mudança topográfica acompanhou também o baixo desempenho dessas cerâmicas nos testes de resistência. Do mesmo modo e, pelo mesmo motivo de insuficiente alteração topográfica, o ácido a 3\% 
foi contraindicado no estudo de Prochnow, Venturini, Grasel, et al. (2018). Além disso, essa concentração também demonstrou aumento da dimensão fractal da peça cerâmica (Prochnow, Venturini, Scherer, et al. 2018). Todos esses motivos fizeram com que a sua utilização fosse indicada sempre sob precaução (Prochnow, Venturini, Grasel, et al. 2018).

Já as concentrações de 5\% e 10\% apresentaram, de acordo com a maioria dos estudos, superfícies que contribuem para a estabilidade adesiva das peças cerâmicas pelo fato de criarem topografias favoráveis ao embricamento do sistema adesivo e do cimento resinoso (Prochnow, Venturini, Grasel, et al. 2018; Prochnow, Venturini, Scherer, et al. 2018; Venturini, Prochnow, May, et al. 2018). A concentração ácida de 5\%, por exemplo, apresentou alterações regulares da superfície em um maior número de estudos (Prochnow, Venturini, Scherer, et al. 2018; Venturini, Prochnow, May, et al. 2015), além de altas cargas de falha por fadiga (Prochnow, Venturini, Scherer, et al. 2018), sendo a opção mais citada entre os estudos incluidos no trabalho. Outras pesquisas, por sua vez, aprovaram os resultados do uso do ácido a 10\%. Uma delas encontrou maiores irregularidades em relação às outras concentrações, o que, além de não promover o enfraquecimento da cerâmica feldspática em comparação ao grupo controle, permitiu uma penetração adequada do cimento resinoso, criando uma camada homogênea de cimento (Venturini, Prochnow, Pereira, et al. 2018).

Apesar de apresentarem os melhores resultados entre os estudos selecionados, o uso do ácido a 5\% e 10\% também apresentou desvantagens em alguns estudos. Apenas dois estudos apontaram menores valores de resistência nos grupos condicionados a 5\%, já que a pesquisa não encontrou uma penetração homogênea da resina nas irregularidades criadas por essa concentração (Missau et al., 2018; Venturini, Prochnow, May, et al. 2018). Malefícios a partir do uso do ácido a 10\%, por sua vez, incluíram baixos valores da carga de falha por fadiga em comparação às concentrações intermediárias (Prochnow, Venturini, Scherer, et al. 2018), um aumento da dimenção fractal, o que pode causar significativamente o decréscimo da rigidez (Prochnow, Venturini, Guilardi, et al. 2018) e a remoção extensa da matriz vítrea, destruindo em excesso os cristais de dissilicato de lítio e produzindo alterações grosseiras de superfície e defeitos com formatos e tamanhos críticos, que funcionaram como precursores de fratura, reduzindo as qualidades mecânicas do material (Prochnow, Venturini, Scherer, et al. 2018). Esses tipos de defeitos topográficos foram apontados em vários estudos como os precursores das fraturas nas cerâmicas, já que a presença de falhas grandes e espaços não preenchidos por cimento ao longo da superfície interna da coroa cerâmica pode aumentar o estresse interno. $\mathrm{O}$ ar presente na área não preenchida pelo cimento resinoso pode aumentar a susceptibilidade de início desse tipo de falha (Missau et al., 2018; Prochnow 2018b; Venturini, Prochnow, May, et al. 2018; Venturini, Prochnow, Pereira, et al. 2018). Diferentemente da camada de cimento que, quando homogênea, promove maior resistência à fratura por preencher corretamente os defeitos das restaurações cerâmicas (Missau et al., 2018).

Ainda que todos os estudos tenham demonstrado as diferentes mudanças topográficas promovidas nas superfícies condicionadas e suas influências nas características mecânicas e adesivas das cerâmicas, 40\% dos artigos não encontraram diferenças significativas na força flexural a partir da mudança na concentração do ácido (Missau et al., 2018; Prochnow, Venturini, Guilardi, et al. 2018; Venturini, Prochnow, May, et al. 2018). Esses estudos atribuem as falhas encontradas durante os testes não apenas à concentração ácida mas, também, à combinação dos mais diversos fatores que, junto com ela, são responsáveis pela adesão adequada das peças cerâmicas. Entre esses fatores temos, por exemplo, o tempo de condicionamento (Miranda et al., 2019), o tipo da cerâmica vítrea (Prochnow, Venturini, Guilardi, et al. 2018), a viscosidade do cimento (Prochnow, Venturini, Scherer, et al. 2018), o papel do agente de união (Venturini, Prochnow, Rambo, et al. 2015), e o modo de fabricação das peças cerâmicas pelo sistema CAD/CAM, as quais apresentaram irregularidades exacerbadas e predominantes na superfície, fator que foi muito influente para as falhas encontradas (Missau et al., 2018; Prochnow, Venturini, Guilardi, et al. 2018). Aqueles estudos que, inclusive, encontraram diferenças significativas em seus resultados a partir da variação das concentrações, também correlacionam os resultados com esses variados fatores, já que os mesmos 
podem influenciar nas vantagens e desvantagens de cada concentração, otimizando-as ou agravando-as (Prochnow, Venturini, Grasel, et al. 2018; Venturini, Prochnow, Rambo, et al. 2015).

Os estudos utilizados apresentaram como viéses o fato das pesquisas in vitro inviabilizarem a análise de variáveis presentes na rotina clínica, como exposição a fluidos e a degradação mecânica promovida pela mastigação (Missau et al., 2018; Prochnow, Venturini, Scherer, et al. 2018). Também foi apontado a ausência de envelhecimento nas peças (Venturini, Prochnow, May, et al. 2018) e a hidrólise da aderência química nas interfaces (Venturini, Prochnow, Pereira, et al. 2018). Além disso, os testes mecânicos, em sua maioria, permitem a análise de resistência a cargas que incidem apenas em uma única direção (axial), o que não condiz com o ambiente bucal (Missau et al., 2018; Prochnow, Venturini, Guilardi, et al. 2018; Venturini, Prochnow, May, et al. 2015; Venturini, Prochnow, May, et al. 2018; Prochnow, Venturini, Grasel, et al. 2018). São necessários, portanto, mais estudos clínicos e laboratoriais com objetivo de abordar uma maior variedade de cerâmicas e, também, contornar esses viéses.

\section{Conclusão}

Diante a revisão sistemática proposta, conclui-se que o ataque com o ácido hidrofluorídrico é um fator imprescindível para uma adesão eficiente das cerâmicas vítreas e as suas diferentes concentrações possuem grande influência nas transformações topográficas das cerâmicas. Sendo assim, diferentes concentrações ácidas influenciam na qualidade de adesão dos diferentes tipos de cerâmicas, apesar de não ser o único fator responsável por ela. Os melhores achados laboratoriais são de autoria dos ácidos hidrofluorídricos nas concentrações de 5 e 10\%. Entretanto, houve uma predominância de resultados positivos diante a concentração de 5\%, tanto nas cerâmicas feldspáticas, quanto nas de dissilicato de lítio.

Levando em consideração os viéses já mencionados, novas pesquisas deveriam preconizar estudos que se aproximem ao máximo das condições clínicas, onde fatores como a umidade e a incidência de forças além das axiais possam ser analisadas. Ao buscar correlacionar um maior número de fatores, a seleção de um protocolo clínico será mais preciso e condizente com a realidade clínica.

\section{Referências}

Colombo, L.; Gómez, F. \& Goes, M. (2019). Bond Strength of CAD/CAM Restorative Materials Treated with Different Surface Etching Protocols. The Journal of Adhesive Dentistry, 21(4), 307-317. https://doi.org/10.3290/j.jad.a42931

Dennerlein, K., Kiesewetter, F., Kilo, S., Jäger, T., Göen, T., Korinth, G., \& Drexler, H. (2016). Dermal absorption and skin damage following hydrofluoric acid exposure in an ex vivo human skin model. Toxicology Letters, 248, 25-33. https://doi.org/10.1016/j.toxlet.2016.02.015

Gómes, F.; Dibb, R. \& Goes, M. (2018) Effect of Acid Etching on Tridimensional Microstructure of Etchable CAD/CAM Materials. Dental Materials, 34, 944-955. https://doi.org/10.1016/j.dental.2018.03.013

Guyatt, G. H., Oxman, A. D., Vist, G. E., Kunz, R., Falck-Ytter, Y., Alonso-Coello, P., \& Schünemann, H. J. (2008). GRADE: an emerging consensus on rating quality of evidence and strength of recommendations. Bmj, 336(7650), 924-926.

Howick, J., Chalmers, I., Glasziou, P., Greenhalgh, T., Heneghan, C., Liberati, A., \& Thornton, H. (2011). The 2011 Oxford CEBM evidence levels of evidence (introductory document). Oxford Center for Evidence Based Medicine

Medeiros, A. K. B., Campos, M. F. T. P., Costa R. S. G. S., Melo, L. A., Barbosa, G. A. S., Carreiro, A. F. P. (2019) Improvement in quality of life of elderly edentulous patients with new complete dentures: A systematic review. The International Journal of Prosthodontics, 32, 272-277. https://doi.org/10.11607/ijp.6075

Melo, L. A., Lima, C. M., Ferrarez, L. L., Silva, D. N., Leite, F. P. P. (2020). Precisão da adaptação de próteses totais confeccionadas pela tecnologia CAD/CAM: Uma revisão sistemática. Research, Society and Development, 9 (10), 1-18. http://dx.doi.org/10.33448/rsd-v9i10.9154

Miranda, J. S., Monteiro, J. B., Silva, P. N. F., Valera, M. C., Bresciani, E., \& Melo, R. M. (2020). Can different etching protocols change the properties of a hybrid ceramic? General dentistry, 20-25. https://agd.org/generaldentistry

Missau, T., Venturini, A. B., Pereira, G. K. R., Prochnow, C., Valandro, L. F., \& Rippe, M. P. (2018). Fatigue failure load of restored premolars: effect of etching the intaglio surface of ceramic inlays with hydrofluoric acid at different concentrations. Operative dentistry, 43 (2), 81-91. https://doi.org/10.2341/16$345-\mathrm{L}$ 
Moher, D., Liberati, A., Tetzlaff, J., Altman, D. G., \& Prisma Group. (2009). Preferred reporting items for systematic reviews and meta-analyses: the PRISMA statement. PLoS med, 6(7), e1000097.

Mokhtarpour, F.; Alaghehmand, H. \& Khafri, S. (2017) Effect of Hydrofluoric Acid Surface Treatments on Micro-Shear Bond Strength of CAD/CAM ceramics. Electronic Physician., 9(10), 5487-5493. https://dx.doi.org/10.19082/5487

Moura, D. M. D., Araújo, A. M. M. D., Souza, K. B. D., Veríssimo, A. H., \& Tribst, J. P. M. (2020). Hydrofluoric acid concentration, time and use of phosphoric acid on the bond strength of feldspathic ceramics. Brazilian Oral Research, 34(18), 1-10. https://doi.org/10.1590/1807-3107bor-2020.vol34.0018

Moura, D. M. D., Carvalho, I. H. G., Silva, S. E. G., Araújo, G. M., Vila-Nova, T. E. L., Silva, N. R., Souza, K. B., Miranda, L. M. \& Souza, R. O. A. (2019) Cimentação Adesiva em Cerâmicas Vítrias: condicionamento e limpeza pós condicionamento: Parte II. Prótese News, 6(5), 578-590.

Özcan, M.; Allahbeickaraghi, A. \& Dündar, M. (2012) Possible hazardous effects of hydrofluoric acid and recommendations for treatment approach: a review. Clinical Oral Investigation, 16, 15-23. https://doi.org/10.1007/s00784-011-0636-6

Pereira A.S. $\quad$ et al. (2018). Metodologia da $\quad$ uAB/NTE/UFSM. https://repositorio.ufsm.br/bitstream/handle/1/15824/Lic_Computacao_Metodologia-Pesquisa-Cientifica.pdf?sequence=1 .

Prochnow, C., Pereira, G., Venturini, A., Scherer, M., Rippe, M., Bottino, M., Kleverlaan, C. \& Valandro, L. (2018) How Does Hydrofluoric Acid Etching Affect the Cyclic Load-to-Failure of Lithium Disilicate Restorations? Journal of the Mechanical Behavior of Biomedical Materials, 87, 306-311. https://doi.org/10.1016/j.jmbbm.2019.07.040

Prochnow, C., Venturini A., Grasel, R, Gundel, A., Bottino, M. \& Valandro, L. (2018). Adhesion to a Lithium Disilicate Glass Ceramic Etched with Hudrofluoric Acid at Distinct Concentrations. Brazilian Dental Journal, 29(5), 492-499. https://dx.doi.org/10.1590/0103-6440201802080

Prochnow, C., Venturini, A. B., Guilardi, L. F., Pereira, G. K. R., Burgo, T. A. L., Bottino, M. C., Kleverlaan, C. J. \& Valandro, L. F. (2018). Hydrofluoric acid concentrations: effect on the cyclic load-to-failure of machined lithium disilicate restorations. Dental Materials, 34(9), 255-263. https://doi.org/10.1016/j.dental.2018.06.028

Puppin-Rontani, J., Sundfeld, D., Costa, A. R., Correr, A. B., Puppin-Rontani, R. M., Borges, G. A., Sinhoreti M. A. C. \& Correr-Sobrinho, L. (2017). Effect of hydrofluoric acid concentration and etching time on bond strength to lithium disilicate glass ceramic. Operative Dentistry, 42(6), 606-615. https://doi.org/10.2341/16-215-L

Saracoglu, A., Özcan, M., Kumbuloglu, O., \& Turkun, M. (2011). Adhesion of resin composite to hydrofluoric acid-exposed enamel and dentin in repair protocols. Operative dentistry, 36(5), 545-553. https://doi.org/10.2341/10-312-L

Souza, R. \& Bottino, M.A. (2018) Cimentação Adesiva em Próteses Cerâmicas: O Que Realmente eu Preciso Saber? Prótese News, 5(5), 516-531.

Sudré, J. P., Salvio, L. A., Baroudi, K., Sotto-Maior, B. S., Melo-Silva, C. L., \& Assis, N. M. S. P. (2020). Influence of Surface Treatment of Lithium Disilicate on Roughness and Bond Strength. The International Journal od Prosthodontics, 33, 212-216. https://doi.org/10.11607/ijp.6453

Sundfeld Neto, D., Naves, L. Z., Costa, A. R., Correr, A. B., Consani, S., Borges, G. A., \& Correr-Sobrinho, L. (2015). The effect of hydrofluoric acid concentration on the bond strength and morphology of the surface and interface of glass ceramics to a resin cement. Operative dentistry, 40(5), 470-479. https://doi.org/10.2341/14-133-L

Tribst, J. P. M., Dal Piva, A. M. D. O., Lopes, G. C., Borges, A. L. S., Bottino, M. A., Özcan, M., \& de Melo, R. M. (2020). Biaxial flexural strength and Weilbull characteristics of adhesively luted hybrid and reinforced CAD/CAM materials to dentin: effect of self-etching ceramic primer versus hydrofluoric acid etching. Journal of Adhesion Science and Technology, 34(12), 1253-1268. https://doi.org/10.1080/01694243.2019.1710991

Venturini, A. B., Prochnow, C., May, L. G., Bottino, M. C., \& Valandro, L. F. (2015). Influence of hydrofluoric acid concentration on the flexural strength of a feldspathic ceramic. Journal of the Mechanical Behavior of Biomedical Materials, 48, 241-248. http://dx.doi.org/10.1016/j.jmbbm.2015.03.025

Venturini, A. B., Prochnow, C., May, L. G., Kleverlaan, C. J., \& Valandro, L. F. (2018). Fatigue failure load of feldspathic ceramic crowns after hydrofluoric acid etching at different concentrations. The Journal of prosthetic dentistry, 119(2), 278-285. https://doi.org/10.1016/j.prosdent.2017.03.021

Venturini, A. B., Prochnow, C., Pereira, G. K., Werner, A., Kleverlaan, C. J., \& Valandro, L. F. (2018). The effect of hydrofluoric acid concentration on the fatigue failure load of adhesively cemented feldspathic ceramic discs. Dental Materials, 34(4), 667-675. https://doi.org/10.1016/j.dental.2018.01.010

Venturini, A. B., Prochnow, C., Rambo, D., Gundel, A., \& Valandro, L. F. (2015). Effect of hydrofluoric acid concentration on resin adhesion to a feldspathic ceramic. The Journal of Adhesive Dentistry, 17(4), 313-200. https://doi.org/10.3290/j.jad.a34592

Veríssimo, A. H., Moura, D. M. D., Tribst, J. P. M., Araújo, A. M. M. D., \& Leite, F. P. P. (2019). Effect of hydrofluoric acid concentration and etching time on resin-bond strength to different glass ceramics. Brazilian oral research, 33, 1-11. https://doi.org/10.1590/1807-3107bor-2019.vol33.0041

Vila-Nova, T., Silva, N. R., Moura, D. M. D. M., Araújo, G. M., Miranda, L. M., Carvalho, I. H. G., Souxa, K. B., Silva, S. E. G. \& Souza, R. O. A. (2019) Cimentação Adesiva em Cerâmicas Vítrias: condicionamento e limpeza pós condicionamento: Parte I. Prótese News, 6 (5), 578-590. 